\title{
RANGE AND DOA ESTIMATION OF POLARIZED NEAR-FIELD SIGNALS USING FOURTH-ORDER STATISTICS
}

\author{
Baha A. Obeidat, Yimin Zhang, and Moeness G. Amin \\ Center for Advanced Communications \\ Villanova University, Villanova, PA 19085, USA \\ E-mail: \{baha.obeidat,yimin.zhang, moeness.amin\}@villanova.edu
}

\begin{abstract}
An enhanced technique for estimating the range and directionof-arrival (DOA) of narrow-band near-field sources is presented. This technique utilizes fourth-order cumulants of the received signal across an array of two orthogonally polarized sensors. It is shown that the incorporation of the source polarization in an ESPRIT-based angle and range estimation technique provides improved performance over the case where the polarization information is absent in the problem formulation.
\end{abstract}

\section{INTRODUCTION}

Most of the research in array processing has been focused on far-field signals and is based on the assumptions that the source signals are located relatively far from the receiver. However, the far-field assumption of plane wavefronts no longer holds and the typical steering vector characterized by the source DOA is not applicable. The consideration of the curvature of the propagating signal waves allows for the ability of range estimation [1].

Polarization diversity has been proven useful in wireless communications and various types of radar systems. Polarization has also been incorporated in array antennas for improved estimation of far-field signal parameters, including direction finding $[2,3,4,5]$.

In this paper, a novel signal model for near-field sources is presented incorporating the source polarization information. Using this model a polarimetric fourth-order statisticsbased ESPRIT-like algorithm is developed for closed-form DOA and range estimation. It is shown that the use of polarimetric information can significantly improve the estimation accuracy of the source parameters.

\section{SIGNAL MODEL}

\subsection{Geometry}

Consider a linear equi-spaced array of $2 N_{x}$ dual-polarization sensors placed on a flat plane in a two-dimensional surface. Assume that the sensor position errors are negligible and

This work was supported in part by the ONR under Grant No. N00014-98-1-0176 and DARPA under Grant No. MDA97202-1-0022. The content of the information does not necessarily reflect the position or policy of the Government, and no official endorsement should be inferred. the gain and phase of all sensors and corresponding data acquisition equipment are accurately matched. We consider a narrow-band array, i.e., the reciprocal of the bandwidth of any signals received is large compared with the propagation delay across the array. The wavelength of all sources received is $\lambda$. Let $d$ be the interelement spacing of the sensors, which are located along the $x$-axis and indexed $-N_{x}+1,-N_{x}+2, \ldots, 0,1, \ldots, N_{x}$ from left to right. We assume that $d \leq \lambda / 4$. The array configuration is shown in Fig. 1.

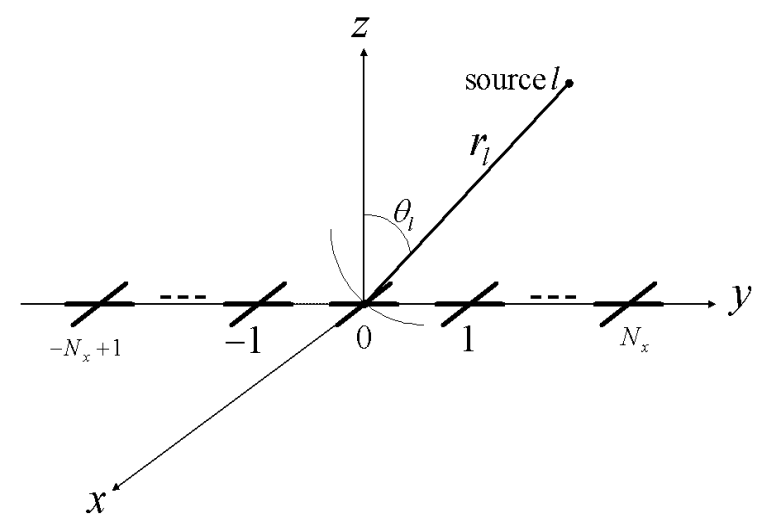

Figure 1: Array geometry.

\subsection{Signal model}

For a transverse electromagnetic (TEM) wave propagating to the array, the electric field can be described as

$$
\overrightarrow{\mathbf{E}}=E_{\phi} \hat{\phi}+E_{\theta} \hat{\theta},
$$

where $\hat{\phi}$ and $\hat{\theta}$ are the spherical unit vectors along the azimuth and elevation angles $\phi$ and $\theta$, respectively. For simplicity, it is assumed that the source signal is in the $y-z$ plane perpendicular to that of the array which is located in the $x$ - $y$ plane. Then, $\phi=90, \hat{\phi}=-\hat{x}$, and

$$
\overrightarrow{\mathbf{E}}=-E_{\phi} \hat{x}+E_{\theta} \hat{\theta}=-E_{\phi} \hat{x}+E_{\theta} \cos (\theta) \hat{y}+E_{\theta} \cos (\theta) \hat{z},
$$

with $\hat{y}$ and $\hat{z}$ representing the unit vectors along the $y$ and $z$ directions, respectively. A polarized signal can be described as

$$
E_{\phi}=E \cos (\gamma), \quad E_{\theta}=E \sin (\gamma) e^{j \eta} .
$$


where $\gamma$ and $\eta$ denote the magnitude ratio and the phase between the two polarization components. Therefore, Eq. (2) can be written as

$$
\overrightarrow{\mathbf{E}}=E\left(-\cos (\gamma) \hat{x}+\cos (\theta) \sin (\gamma) e^{j \eta} \hat{y}+\cos (\theta) \hat{z}\right)
$$

The signal component in the $\hat{z}$ direction is eliminated using the specific orientation of the cross-polarized array of Fig. 1. It is noted that, in the near-field environment, $\theta$ in the above equation varies at each of the $2 N_{x}$ sensors. It is straightforward to show that the DOA of the $l$ th source signal at sensor $m$ is described by

$$
\theta_{m l}=\sin ^{-1}\left(\frac{r_{l} \sin \left(\theta_{l}\right)-m d}{\sqrt{r_{l}^{2}+m^{2} d^{2}-2 r_{l} m d \sin \left(\theta_{l}\right)}}\right) .
$$

The pair of variables $\left(\theta_{l}, r_{l}\right)$ denotes the DOA and range of source $l$ at the reference sensor, $m=0$. These variables are unknown and need to be estimated.

The received signal at sensor $m$ for polarizations $x$ and $y$ can be approximated as [1]

$$
u_{m}^{[x]}(t)=-\sum_{l=1}^{N_{s}} s_{l}(t) \cos \left(\gamma_{l}\right) e^{j\left(\omega_{l} m+\phi_{l} m^{2}\right)}+n_{m}^{[x]}(t),
$$

$u_{m}^{[y]}(t)=\sum_{l=1}^{N_{s}} s_{l}(t) \cos \left(\theta_{m l}\right) \sin \left(\gamma_{l}\right) e^{j \eta_{l}} e^{j\left(\omega_{l} m+\phi_{l} m^{2}\right)}+n_{m}^{[y]}(t)$,

respectively, where $n_{m}^{[i]}(t), i=x, y$ is the noise component for polarization $i$ at the $m$ th sensor and $s_{l}(t)$ is the $l$ th source signal. The parameters $\omega_{l}$ and $\phi_{l}$ are functions of the DOA $\theta_{l}$, the range $r_{l}$, and the wavelength $\lambda$ of source $l$, and are defined as

$$
\omega_{l}=-2 \pi \frac{d}{\lambda} \sin \left(\theta_{l}\right), \quad \phi_{l}=\pi \frac{d^{2}}{\lambda r_{l}} \cos ^{2}\left(\theta_{l}\right) .
$$

In vector format, Eqs. (6) and (7) can be written as

$$
\mathbf{u}^{[i]}(t)=\mathbf{B}^{[i]} \mathbf{s}^{[i]}(t)+\mathbf{n}^{[i]}(t), \quad i=x, y
$$

with

$$
\begin{aligned}
\mathbf{u}^{[i]}(t) & =\left[u_{-N_{x}+1}^{[i]}(t), u_{-N_{x}+2}^{[i]}(t), \ldots, u_{N_{x}}^{[i]}(t)\right]^{T} \\
\mathbf{s}^{[x]}(t) & =-\left[s_{1}(t) \cos \left(\gamma_{1}\right), \ldots, s_{N_{s}}(t) \cos \left(\gamma_{N_{s}}\right)\right]^{T} \\
\mathbf{s}^{[y]}(t) & =\left[s_{1}(t) \sin \left(\gamma_{1}\right) e^{j \eta_{1}}, \ldots, s_{N_{s}}(t) \sin \left(\gamma_{N_{s}}\right) e^{j \eta_{N_{s}}}\right]^{T} \\
\mathbf{n}^{[i]}(t) & =\left[n_{-N_{x}+1}^{[i]}(t), n_{-N_{x}+2}^{[i]}(t), \ldots, n_{N_{x}}^{[i]}(t)\right]^{T} .
\end{aligned}
$$

The columns of the polarization-dependent $2 N_{x} \times N_{s}$ steering matrices $\mathbf{B}^{[x]}$ and $\mathbf{B}^{[y]}$ are

$$
\begin{aligned}
\mathbf{b}_{l}^{[x]}= & {\left[e^{j\left(-N_{x}+1\right) \omega_{l}+j\left(-N_{x}+1\right)^{2} \phi_{l}}, \ldots, 1,\right.} \\
& \left.e^{j\left(\omega_{l}+\phi_{l}\right)}, \ldots, e^{j\left(N_{x} \omega_{l}+N_{x}^{2} \phi_{l}\right)}\right]^{T} \\
\mathbf{b}_{l}^{[y]}= & {\left[e^{j\left(-N_{x}+1\right) \omega_{l}+j\left(-N_{x}+1\right)^{2} \phi_{l}} \cos \left(\theta_{\left(-N_{x}+1\right) l}\right),\right.} \\
& \ldots, \cos \left(\theta_{0 l}\right), e^{j\left(\omega_{l}+\phi_{l}\right)} \cos \left(\theta_{1 l}\right), \ldots, \\
& \left.e^{j\left(N_{x} \omega_{l}+N_{x}^{2} \phi_{l}\right)} \cos \left(\theta_{N_{x} l}\right)\right]^{T},
\end{aligned}
$$

where the superscript $T$ denotes transpose. Note that in the above formulation, the source polarization appears, in part, in both $\mathbf{B}^{[q]}$ and $\mathbf{s}^{[i]}(t)$.

We make the following assumptions:

[A1] The source signals $s_{l}^{[i]}(t), l=1,2, \ldots, N_{s}$, are independent, non-Gaussian, narrow-band, and stationary processes.

[A2] The noise components, $n_{m}^{[i]}(t), m=-N_{x}+1,-N_{x}$ $+2, \ldots, N_{x}$, are independent and zero-mean Gaussian processes, and are independent of the source signals.

$[$ A3 $]$ The range of the sources are not equal, i.e., $r_{i} \neq r_{j}$ for $i \neq j$.

[A4] The array is uniform and linear with $2 N_{x}$ sensors. The interelement spacing of the array is $d \leq \frac{\lambda}{4}$. Additionally, the number of sources is less than half the number of sensors, i.e., $N_{s}<N_{x}$.

\section{ESTIMATION ALGORITHM}

The $(m, n)$ th element of the polarized fourth-order cumulant matrix $\mathbf{C}_{\mathbf{1}}{ }^{[i j]}$ is defined as

$$
C_{1}^{[i j]}(m, n)=\operatorname{cum}\left\{\left(u_{m}^{[i]}(t)\right)^{*}, u_{m+1}^{[i]}(t),\left(u_{n+1}^{[j]}(t)\right)^{*}, u_{n}^{[j]}(t)\right\}
$$

where $i, j=x, y$ and $0 \leq m, n \leq N_{x}-1$. Using the multilinearity property of cumulants and the assumptions A1 $\mathrm{A} 4$, we can write $\mathbf{C}_{\mathbf{1}}{ }^{[x x]}=\left[C_{1}^{[x x]}(m, n)\right]$ as

$$
\mathbf{C}_{\mathbf{1}}{ }^{[x x]}=\mathbf{A} \mathbf{C}_{\mathbf{4 s}}^{[x x]} \mathbf{A}^{H} \text {. }
$$

where $\mathbf{A}$ is an $N_{x} \times N_{s}$ matrix with its $l$ th column given by

$$
\mathbf{a}_{l}=\left[1, e^{j 2 \phi_{l}}, \ldots, e^{j 2\left(N_{x}-1\right) \phi_{l}}\right]^{T},
$$

and the matrix $\mathbf{C}_{4 \mathbf{s}}^{[i j]}$ of size $N_{s} \times N_{s}$ is diagonal and its $k$ th diagonal element is the cross- or auto-polarized signal kurtosis of the $k$ th signal. For different polarizations, $\mathbf{C}_{4 \mathbf{s}}^{[x x]}=\mathbf{P C}_{4 \mathbf{s}} \mathbf{P}, \mathbf{C}_{4 \mathbf{s}}^{[x y]}=\mathbf{P C}_{4 \mathbf{s}} \mathbf{Q}, \mathbf{C}_{4 \mathbf{s}}^{[y x]}=\mathbf{Q C}_{4 \mathbf{s}} \mathbf{P}$ and $\mathbf{C}_{4 \mathbf{s}}^{[y y]}=\mathbf{Q C}_{4 \mathbf{s}} \mathbf{Q}$, where

$$
\begin{aligned}
\mathbf{P} & =\operatorname{diag}\left[\cos ^{2}\left(\gamma_{1}\right), \ldots, \cos ^{2}\left(\gamma_{N_{s}}\right)\right] \\
\mathbf{Q} & =\operatorname{diag}\left[\sin ^{2}\left(\gamma_{1}\right), \ldots, \sin ^{2}\left(\gamma_{N_{s}}\right)\right] \\
\mathbf{C}_{4 \mathbf{s}} & =\operatorname{diag}\left[c_{4 s_{1}}, c_{4 s_{2}}, \ldots, c_{4 s_{N_{x}}}\right] \\
c_{4 s_{l}} & =\operatorname{cum}\left\{s_{l}^{*}(t), s_{l}(t), s_{l}^{*}(t), s_{l}(t)\right\} .
\end{aligned}
$$

Table 1 describes the matrix product formulation similar to (14) for $x y, y x$ and $y y . \overline{\mathbf{A}}$ is the steering matrix of size $N_{x} \times N_{s}$ associated with the $y$ polarization. Assuming that the $\cos \left(\theta_{m l}\right)$ term in $(7)$ is constant across all sensors, i.e. $\cos \left(\theta_{m l}\right)=\cos \left(\theta_{0 l}\right)$, then one can define the columns of matrix $\overline{\mathbf{A}}$ as

$$
\overline{\mathbf{a}}_{l}=\cos ^{2}\left(\theta_{0 l}\right)\left[1, e^{j 2 \phi_{l}}, \ldots, e^{j 2\left(N_{x}-1\right) \phi_{l}}\right]^{T} .
$$

It is noteworthy that relaxing the above assumption does not impede the performance of the proposed polarimetric approach.

Table 1: Cross-polarized cumulant matrices

\begin{tabular}{lc}
\hline $\mathbf{C}_{\mathbf{1}}{ }^{[i j]}$ & Cumulant matrix \\
\hline $\mathbf{C}_{\mathbf{1}}{ }^{[x x]}$ & $\mathbf{A} \mathbf{C}_{\mathbf{4}}^{[x]} \mathbf{A}^{H}$ \\
$\mathbf{C}_{\mathbf{1}}{ }^{[x y]}$ & $\mathbf{A} \mathbf{C}_{\mathbf{4}}^{[x y]} \overline{\mathbf{A}}^{H}$ \\
$\mathbf{C}_{\mathbf{1}}{ }^{[y x]}$ & $\overline{\mathbf{A}} \mathbf{C}_{\mathbf{4}}^{[y x]} \mathbf{A}^{H}$ \\
$\mathbf{C}_{\mathbf{1}}{ }^{[y y]}$ & $\overline{\mathbf{A}} \mathbf{C}_{\mathbf{4 s}}^{[y y]} \overline{\mathbf{A}}^{H}$ \\
\hline
\end{tabular}


Using different sensor lags, one can define other fourthorder polarized cumulant matrices,

$$
\begin{aligned}
& C_{2}{ }^{[i j]}(m, n)=\operatorname{cum}\left\{\left(u_{m-1}^{[i]}(t)\right)^{*}, u_{m}^{[i]}(t),\left(u_{-n}^{[j]}(t)\right)^{*}, u_{1-n}^{[j]}(t)\right\} \\
& C_{3}{ }^{[i j]}(m, n)=\operatorname{cum}\left\{\left(u_{m}^{[i]}(t)\right)^{*}, u_{m+1}^{[i]}(t),\left(u_{-n}^{[j]}(t)\right)^{*}, u_{1-n}^{[j]}(t)\right\} \\
& C_{4}{ }^{[i j]}(m, n)=\operatorname{cum}\left\{\left(u_{m-1}^{[i]}(t)\right)^{*}, u_{m}^{[i]}(t),\left(u_{n+1}^{[j]}(t)\right)^{*}, u_{n}^{[j]}(t)\right\} .
\end{aligned}
$$

From Table 2 and the four respective polarized cumulant matrices of Table 1, other cumulant matrices can be described as a function of $\mathbf{A}, \overline{\mathbf{A}}, \mathbf{C}_{\mathbf{4 s}}^{[i j]}, \boldsymbol{\Phi}$ and $\boldsymbol{\Omega}$. The latter two diagonal matrices are defined in terms of the variables $\omega_{l}$ and $\phi_{l}, l=1,2, \ldots, N_{s}$, introduced in (8), where

$$
\begin{aligned}
& \boldsymbol{\Omega}=\operatorname{diag}\left[e^{-j 2 \omega_{1}}, e^{-j 2 \omega_{2}}, \ldots, e^{-j 2 \omega_{N_{s}}}\right] \\
& \boldsymbol{\Phi}=\operatorname{diag}\left[e^{j 2 \phi_{1}}, e^{j 2 \phi_{2}}, \ldots, e^{j 2 \phi_{N_{s}}}\right]
\end{aligned}
$$

Table 2: Cross-polarized cumulant matrices

\begin{tabular}{lc}
\hline Matrix & Equivalent \\
\hline $\mathbf{C}_{\mathbf{1}}{ }^{[x x]}$ & $\mathbf{A} \mathbf{C}_{4 \mathbf{s}}^{[x x]} \mathbf{A}^{H}$ \\
$\mathbf{C}_{\mathbf{2}}{ }^{[x x]}$ & $\mathbf{A} \mathbf{C}_{\mathbf{4 s}}^{[x x]} \boldsymbol{\Omega}^{H} \mathbf{A}^{H}$ \\
$\mathbf{C}_{\mathbf{3}}{ }^{[x x]}$ & $\mathbf{A} \boldsymbol{\Phi}^{H} \mathbf{C}_{\mathbf{4 s}}^{[x x]} \mathbf{\Omega}^{H} \mathbf{A}^{H}$ \\
$\mathbf{C}_{\mathbf{4}}{ }^{[x x]}$ & $\mathbf{A C}_{\mathbf{4 s}}^{[x x]} \boldsymbol{\Phi}^{H} \mathbf{A}^{H}$ \\
\hline
\end{tabular}

Let $\mathbf{C}$ denote the dual-polarized matrix

$$
\mathbf{C}=\left[\begin{array}{ll}
\mathbf{C}^{[x x]} & \mathbf{C}^{[x y]} \\
\mathbf{C}^{[y x]} & \mathbf{C}^{[y y]}
\end{array}\right]
$$

For $i, j=x, y$, each sub-matrix in (26) can be defined as [7]

$$
\mathbf{C}^{[i j]}=\left[\begin{array}{ccc}
\mathbf{C}_{\mathbf{1}}{ }^{[i j]} & \mathbf{C}_{\mathbf{2}}{ }^{[i j]} & \mathbf{C}_{4}{ }^{[i j]} \\
\left(\mathbf{C}_{\mathbf{2}}{ }^{[i j]}\right)^{H} & \mathbf{C}_{\mathbf{1}}{ }^{[i j]} & \left(\mathbf{C}_{\mathbf{3}}{ }^{[i j]}\right)^{H} \\
\left(\mathbf{C}_{4}{ }^{[i j]}\right)^{H} & \mathbf{C}_{\mathbf{3}}{ }^{[i j]} & \mathbf{C}_{\mathbf{1}}{ }^{[i j]}
\end{array}\right] .
$$

Matrix $\mathbf{C}$ can be conveniently written in a form reminiscent to that used in array processing signal models

$$
\mathbf{C} \simeq \hat{\mathbf{A}} \mathbf{C}_{4 \mathbf{s}} \hat{\mathbf{A}}^{H}
$$

where matrix $\hat{\mathbf{A}}$ is of size $6 N_{x} \times 2 N_{s}$ and given by

$$
\hat{\mathbf{A}}=\left[\begin{array}{l}
\mathbf{A P} \\
\mathbf{A P} \Omega \\
\mathbf{A P} \Phi \\
\overline{\mathbf{A}} \mathbf{Q} \\
\overline{\mathbf{A}} \mathbf{Q} \Omega \\
\overline{\mathbf{A}} \mathbf{Q} \Phi
\end{array}\right]
$$

From (29), it is evident that the polarization diversity doubles the space dimension of the conventional problem formulation.

Performing an eigendecomposition on $\mathbf{C}$ and selecting the eigenvectors corresponding to the largest $N_{s}$ eigenvalues, we define the signal subspace matrix, $\mathbf{E}_{s}=\left[\mathbf{e}_{1}, \mathbf{e}_{2}, \ldots\right.$, $\left.\mathbf{e}_{N_{s}}\right]$, which can be partitioned as

$$
\mathbf{E}_{s}=\left[\left(\mathbf{E}_{1}^{[x]}\right)^{T},\left(\mathbf{E}_{2}^{[x]}\right)^{T},\left(\mathbf{E}_{3}^{[x]}\right)^{T},\left(\mathbf{E}_{1}^{[y]}\right)^{T},\left(\mathbf{E}_{2}^{[y]}\right)^{T},\left(\mathbf{E}_{3}^{[y]}\right)^{T}\right]^{T} .
$$

Define

$$
\mathbf{E}_{1}=\left[\begin{array}{l}
\mathbf{E}_{1}^{[x]} \\
\mathbf{E}_{1}^{[y]}
\end{array}\right], \mathbf{E}_{2}=\left[\begin{array}{l}
\mathbf{E}_{2}^{[x]} \\
\mathbf{E}_{2}^{[y]}
\end{array}\right] \text { and } \mathbf{E}_{3}=\left[\begin{array}{l}
\mathbf{E}_{3}^{[x]} \\
\mathbf{E}_{3}^{[y]}
\end{array}\right]
$$

From the definition of $\hat{\mathbf{A}}$ in (29) and using an ESPRITlike rotational invariance, the subspace spanned by the columns of both $\mathbf{E}_{2}$ and $\mathbf{E}_{3}$ are rotated versions of that spanned by the columns of $\mathbf{E}_{1}$. Therefore, one can define the following two key equations

$$
\mathbf{E}_{2}=\mathbf{E}_{1} \boldsymbol{\Psi} \quad \text { and } \quad \mathbf{E}_{3}=\mathbf{E}_{1} \mathbf{\Upsilon}
$$

where the eigenvalues of the matrices $\boldsymbol{\Psi}$ and $\boldsymbol{\Upsilon}$ are the diagonal elements of $\boldsymbol{\Omega}$ and $\boldsymbol{\Phi}$. In the least-squares sense $[6], \boldsymbol{\Psi}$ and $\boldsymbol{\Upsilon}$ can be estimated as

$$
\boldsymbol{\Psi}=\mathbf{E}_{1}^{\dagger} \mathbf{E}_{2} \quad \text { and } \quad \boldsymbol{\Upsilon}=\mathbf{E}_{1}^{\dagger} \mathbf{E}_{3}
$$

where $\dagger$ represents the pseudo-inverse. An eigendecomposition of the least-squares solution presented in (33) yields closed-form estimates of $\omega_{l}$ and $\phi_{l}$ in (8), and subsequently estimates of $\theta_{l}$ and $r_{l}$.

\section{SIMULATIONS}

To demonstrate the advantages of using dual-polarization array in the underlying range and DOA estimation problem, two sets of simulations are performed; one pertains to the DOA estimation whereas the other considers the range estimation. The receiver array consists of 14 crossed-dipoles (i.e., horizontally and vertically polarized dipoles) with an interelement spacing of $d=\lambda / 4$. Two sources with parameters shown in Table 3 impinge on the array and are modelled as $e^{j \psi_{t}}$, where the phases $\psi_{t}, t=1,2, \ldots, N$, are uniformly distributed in the interval $[0,2 \pi] . N=1000$ samples of the source signals are used. The root mean-square error (RMSE) is plotted versus the input signal-to-noise ratio (SNR) at each of the sensors.

The proposed polarimetric method was compared to the conventional ESPRIT-like method, which does not incorporate the signal polarizations. In the conventional method, there was no decoupling of the polarization variables and the signal received at both polarizations was combined.

Figures 2 and 3 show the RMSE of the range estimates of 100 independent trials at each SNR point for the conventional and the polarimetric methods, respectively. It is evident that the polarimetric method outperforms the conventional method. Furthermore, improved estimates are provided as the source moves closer to the receiver, which is consistent with the behavior of cumulant-based estimation [7].

Figures 4 and 5 show the RMSE of the DOA estimates of 100 independent trials at each SNR point for both methods. Similar to the findings in [7], the angle estimation is not affected by the source range. It is evident from both figures that the polarimetric method is superior to the conventional method. 
Table 3: Signal parameters

\begin{tabular}{lcccc}
\hline & $\begin{array}{c}\text { DOA } \\
\text { (deg.) }\end{array}$ & $\begin{array}{c}\text { range } \\
\text { (in } \lambda)\end{array}$ & $\begin{array}{c}\gamma \\
\text { (deg.) }\end{array}$ & $\begin{array}{c}\phi \\
\text { (deg.) }\end{array}$ \\
\hline source 1 & 20 & 0.5 & 10 & 0 \\
source 2 & 30 & 5.0 & 80 & 0 \\
\hline
\end{tabular}

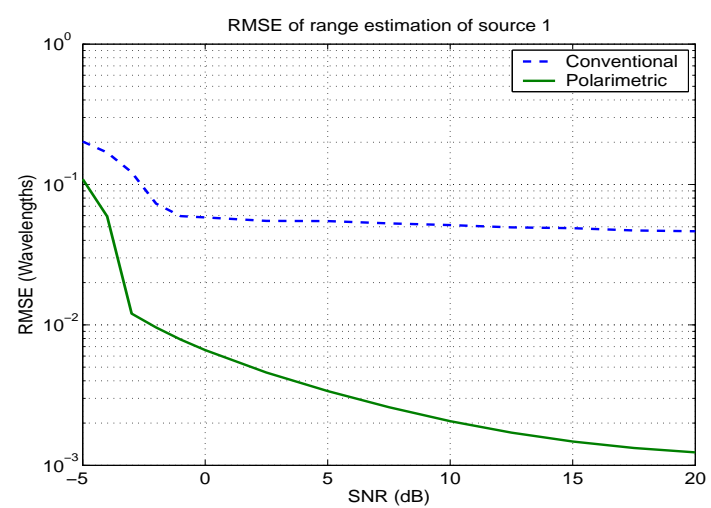

Figure 2: RMSE range of source 1.

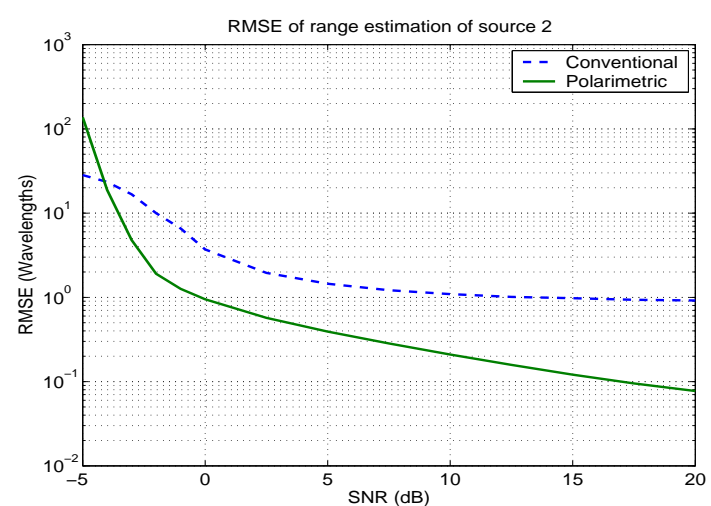

Figure 3: RMSE range of source 2.

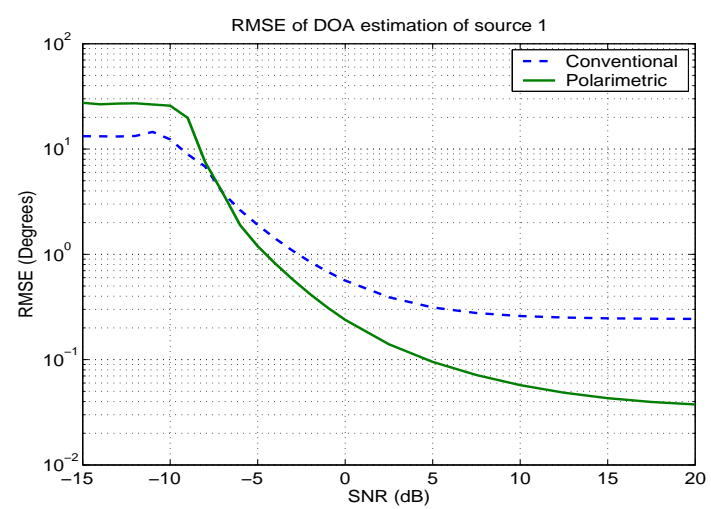

Figure 4: RMSE DOA of source 1.

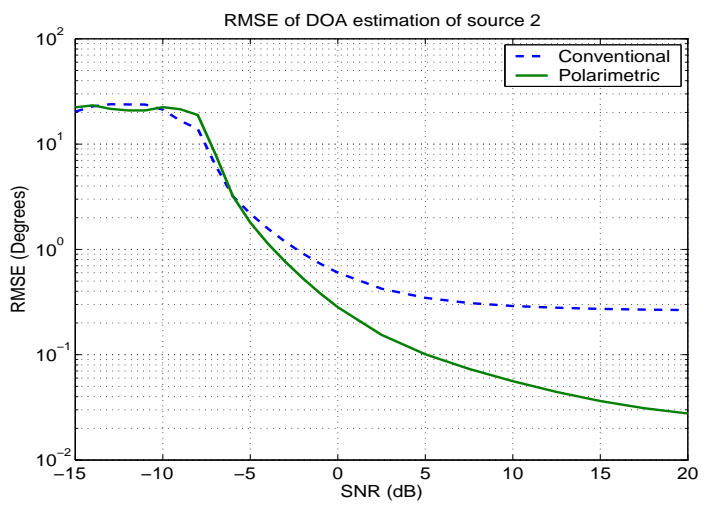

Figure 5: RMSE DOA of source 2.

\section{CONCLUSION}

A technique for DOA and range estimation using crosspolarized multi-antenna array was presented. This technique is based on an ESPRIT-like algorithm and incorporates fourth-order cumulants for improved source parameter estimation of polarized near-field signals. It is evident from the root mean-square error simulations of the source DOA and range parameters that the proposed technique outperforms its non-polarimetric counterpart.

\section{REFERENCES}

[1] R. N. Challa and S. Shamsunder, "High-order subspace-based algorithms for passive localization of near-field sources," Asilomar Conference on Signals, Systems and Computers, Pacific Grove, CA, pp. 777781, Nov. 1995.

[2] E. R. Ferrara and T. M. Parks, "Direction finding with an array of antennas having diverse polarizations," IEEE Trans. Antennas Propagat., vol. 31, pp. 231-236, March 1983.

[3] J. Li and R. J. Compton, "Angle and polarization estimation using ESPRIT with a polarization sensitive array," IEEE Trans. Antennas Propagat., vol. 39, pp. 1376-1383, Sept. 1991.

[4] Y. Zhang, M. G. Amin, and B. A. Obeidat, "Spatial polarimetric time-frequency distributions and applications to direction-of-arrival estimation," SPIE Annual Meeting, vol. 5205, San Diego, CA, Aug. 2003.

[5] B. A. Obeidat, Y. Zhang, and M. G. Amin, "Polarimetric time-frequency ESPRIT," Asilomar Conference on Signals, Systems, and Computers, Pacific Grove, CA, Nov. 2003.

[6] R. Roy and T. Kailath, "ESPRIT-estimation of signal parameters via rotational invariance techniques," IEEE Trans. Acoust., Speech, Signal Processing, vol. 37, pp. 984-995, July 1989.

[7] N. Yuen and B. Friedlander, "Performance analysis of higher order ESPRIT for localization of near-field sources," IEEE Trans. Signal Processing, vol. 46, pp. 709-719, Mar. 1998. 